\section{EPIGENETICS}

\section{Cloning senescence}

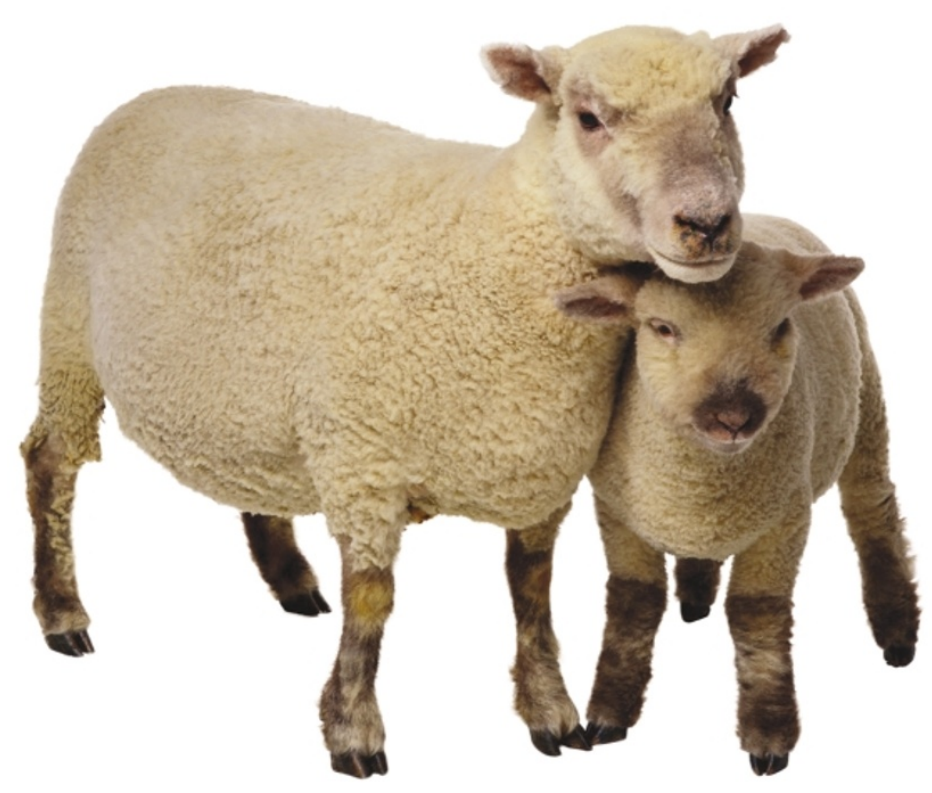

Cloning by nuclear transfer (NT) of adult somatic nuclei into enucleated oocytes involves epigenetic reprogramming to allow the expression of gene programmes appropriate for early development. One of the key questions is whether cellular ageing is reset during reprogramming. It certainly seems to be, at least at the organismal level, given the reports of a relatively normal lifespan for many animals cloned by somatic cell NT. Previous reports have suggested that cattle fetuses cloned from cultured donor cells at, or near, senescence grow into healthy calves and yield fibroblasts with extended lifespans and telomere length relative to cells from agematched control fetuses. Now, John Clark and colleagues revisit this crucial question by carrying out nuclear-transfer experiments in sheep, using cell lines with a range of proliferative capacities. Remarkably, primary cell lines derived from cloned progeny invariably had the same proliferative capacity as the donor cell lines. Furthermore, the actual age of the donor nucleus does not seem to alter the lifespan of the NT clone-derived cell lines.

To assess the rate with which the cloned cells age, the authors turned to the telomeres because they are eroded as the cell divides and, unless replenished, are thought to contribute to cellular senescence once they reach a crucial length. Based on the inverse correlation between telomere erosion rates and proliferative vigour of the cell lines the authors suggest that the rate of telomere shortening determines lifespan.

Importantly, the rates of telomere erosion of the lines derived after NT cloning closely matched those of the parental cultures. These data are consistent with previous reports from cattle, in that telomeres from donor cells near senescence are extended upon NT; however, they do not reproduce the claim that bovine telomeres are extended upon cloning relative to non-cloned controls. These data are also consistent

\title{
Detective work uncovers a new way
}

Most human genetic disorders arise from mutations that disrupt coding sequences or conserved local or distant (although this is much rarer) regulatory regions, including promoters and mRNA processing signals. Tufarelli and colleagues have now extended this list by showing that a genetic disorder $-\alpha$-thalassemia, in this case - can be brought about by transcription of antisense RNA that leads to methylation and gene silencing.

The authors had previously faced a dilemma - they identified a patient with $\alpha$-thalassemia but no corresponding mutation in the coding or regulatory regions of the adult $\alpha 2$-globin gene (HBA2). This patient did, however, carry a deletion in the $\alpha$-globin cluster. Despite the positively acting cis-elements being intact, $H B A 2$ was silenced and its $\mathrm{CpG}$ island was heavily methylated resulting in $\alpha$-thalassemia.

It turned out that the deletion positions a truncated copy of a widely expressed component of the splicing machinery
(LUC7L) next to HBA2. LUC7L is transcribed from the opposite strand to $H B A 2$, and because antisense RNA has been implicated in maternal imprinting and $\mathrm{X}$ inactivation the authors used RT-PCR to look for $L U C 7 L$ transcripts. The results showed that in the deleted globin cluster, transcripts that initiate from the $L U C 7 L$ promoter fail to terminate (the termination site having been removed by the deletion) and extend into the $\mathrm{CpG}$-island region of HBA 2.

To investigate the role of this antisense transcript on the silencing and methylation of $H B A 2$, Tufarelli et al. made a mouse model to mimic the effects of this deletion. They found that, as in their patient, the expression of $\mathrm{Hba}-\mathrm{a} 2$ was abolished in the presence of the antisense transcripts. There was also a strong correlation between the methylation of the $\mathrm{Hba} 2 \mathrm{CpG}$ island and the presence of the antisense transcript.

The authors also established a model of this deletion in embryonic stem (ES) cells.
Working in tissue culture allowed them to determine that methylation was CpGisland-specific and that removing the minimal promoter of $H B A 2$ silenced the gene but did not induce methylation further indicating that antisense transcription was necessary for the methylation and subsequent silencing. Finally, the authors showed that other antisense RNAs that are complementary to the $H B A 2$ promoter also resulted in methylation and silencing, which indicated that the mechanism is not specific to the aberrant $L U C 7 L$ transcript.

So, although the authors do not propose an exact mechanism by which antisense RNA brings about methylation and silencing, they point out that given their example and what we know about $\mathrm{X}$ inactivation and imprinting, regulation of gene expression by antisense RNA might be a general natural phenomenon. No doubt Tufarelli et al. will use their mouse model and the ES system that they have set up to look into the mechanism of this process more closely.

(4) References and links Magdalena Skipper ORIGINAL RESEARCH PAPER Tufarelli, C. et al. Transcription of antisense RNA leading to gene silencing and methylation as a novel cause of human genetic disease. Nature Genet. 34, 157-165 (2003) 